\title{
Cárie dentária entre escolares do meio rural de Itaúna (MG), Brasil
}

\author{
Mauro Henrique Nogueira Guimarães de Abreu, ${ }^{1}$ \\ Isabela Almeida Pordeus ${ }^{2}$ e Celina Maria Modena ${ }^{3}$
}

Como citar Abreu MHNG de, Pordeus IA, Modena CM. Cárie dentária entre escolares do meio rural de Itaúna (MG), Brasil. Rev Panam Salud Publica. 2004;16(5):334-44.

RESUMO Objetivo. Investigar a presença de cárie e sua associação com variáveis socioeconômicas entre escolares do meio rural de Itaúna, Estado de Minas Gerais, em 2002.

Método. Realizou-se um levantamento epidemiológico no qual 476 escolares entre 4 e 15 anos foram submetidos a um exame dentário. Todos os exames foram feitos pelo mesmo examinador, que utilizou a metodologia da Organização Mundial da Saúde. O levantamento das variáveis socioeconômicas (renda familiar mensal, anos de estudo formal do pai e da mãe, ocupação do pai e da mãe, origem da água consumida, sexo e idade da criança) foi feito a partir de entrevistas com os pais dos escolares.

Resultados. Mais de três quartos dos pais recebiam até 360 reais (aproximadamente 153 dólares) por mês e relataram ter freqüentado a escola por, no máximo, 4 anos. Foram identificados como livres de cárie em ambas as dentições $17,86 \%$ dos escolares. O número médio de dentes permanentes cariados, perdidos e restaurados e de dentes decíduos cariados, com extração indicada e obturados foi de 0,94 $( \pm 1,55)$ e 4,00 ( $\pm 3,46)$, respectivamente. A análise multivariada mostrou que as crianças com 7 e 8 anos cuja renda familiar mensal era superior a $R \$ 280$ tinham 2,602 (IC95\%: 1,004 a 6,745) e 2,854 (IC95\%: 1,044 a 7,799) vezes mais chance de apresentar cárie, respectivamente, na dentição decídua e em ambas as dentições. A escolaridade da mãe superior a 3 anos aumentou em 2,813 (IC95\%: 1,221 a 6,480) vezes a chance de o escolar com 7 e 8 anos apresentar cárie na dentição permanente.

Conclusões. Tendo em vista os resultados - especialmente em relação à dentição decídua é urgente a implementação de estratégias coletivas em saúde bucal direcionadas a uma faixa etária precoce. Os resultados sugerem que, em populações com privações econômicas, como é o caso do meio rural de Itaúna, um pouco mais de renda e educação podem significar maior acesso a hábitos que originam cárie.

Palavras-chave Escolaridade, índice $\mathrm{CPO}$, renda familiar.

Universidade Estadual de Montes Claros, Departamento de Odontologia, Montes Claros (MG); e Centro Universitário Newton Paiva, Curso de Odontologia, Belo Horizonte (MG), Brasil. Enviar correspondência para este autor no seguinte endereço: Rua Nascimento Gurgel 750/501, CEP 30430-340, Belo Horizonte, MG, Brasil. Telefone: +55-31-3313-2384; e-mail: mauroh@teacher.com

2 Universidade Federal de Minas Gerais (UFMG), Departamento de Odontopediatria e Ortodontia, Belo Horizonte (MG), Brasil.
A cárie dentária ainda representa o principal problema de saúde bucal coletiva no Brasil, apesar de ter sido registrada uma tendência para a dimi-

\footnotetext{
3 UFMG, Departamento de Medicina Veterinária Preventiva.
}

nuição de sua prevalência na década de $1990(1,2)$. Entretanto, a maioria dos estudos, incluindo os dois levantamentos realizados e divulgados pelo Ministério da Saúde até 2002 (1, 3), foi desenvolvida em meio urbano. $\mathrm{O}$ conhecimento sobre as condições dentárias de populações rurais é pequeno, 
embora essas populações representem quase $20 \%$ dos habitantes do país (4). Os restritos estudos já publicados sobre saúde bucal de populações rurais no Brasil são, em sua maioria, descritivos, e apresentam importantes problemas metodológicos (5). Localizou-se apenas um trabalho que utiliza metodologia epidemiológica analítica (6). Tais estudos mostram, na década de 1980 (7-12), uma prevalência maior de cárie em relação a populações urbanas. Na década de 1990 (13-17), observa-se uma redução na prevalência da cárie no meio rural. No entanto, um estudo realizado na zona rural de Itamonte, no Estado de Minas Gerais, sugere que o quadro de saúde bucal ainda é bastante precário (18).

Da mesma forma, existe uma lacuna quanto ao perfil socioeconômico da população rural no Brasil. Um maior conhecimento acerca desse aspecto é fundamental, já que há fortes evidências, na literatura nacional e internacional, da associação entre os fatores sociais e a ocorrência de cárie (3, 19-27).

Assim, o objetivo do presente estudo foi determinar, a partir de levantamento epidemiológico, a prevalência de cárie dentária nos escolares do meio rural de Itaúna, no Estado de Minas Gerais. Além disso, o estudo buscou relacionar a experiência de cárie com o perfil socioeconômico, definido a partir das variáveis renda familiar mensal, anos de estudo formal do pai e da mãe, ocupação do pai e da mãe, origem da água consumida e sexo e idade da criança.

\section{MATERIAIS E MÉTODOS}

Itaúna, no Estado de Minas Gerais, tem uma população de 76862 habitantes, dos quais $5092(6,62 \%)$ residem no meio rural (4). De acordo com dados da prefeitura, todas as crianças do meio rural estão matriculadas no ensino fundamental público. Existem aproximadamente 40 comunidades rurais no município, mas apenas oito têm escolas municipais que oferecem ensino infantil e as quatro primeiras séries do ensino fundamental. As crianças residentes em comunidades não contempladas com escola são transportadas até a escola mais próxima pelo poder público local (28).

A assistência à saúde bucal é feita pelo Sistema Único de Saúde (SUS), que realiza atendimento individual de atenção básica, geralmente uma vez ao ano, aos escolares e à comunidade do meio rural. O programa odontológico do meio rural envolve, ainda, entrega de escovas e dentifrícios e orientação de escovação para os escolares (28). A população-alvo do estudo foi composta por todos os 503 alunos de educação infantil e das quatro primeiras séries do ensino fundamental do $\mathrm{Mu}$ nicípio de Itaúna. A lista dos alunos regularmente matriculados foi fornecida pela Secretaria Municipal de Educação. O estudo foi aprovado pelo Comitê de Ética em Pesquisa da Universidade Federal de Minas Gerais (UFMG).

\section{Coleta de dados}

Foram realizados contatos iniciais com a prefeitura para que o estudo fosse viabilizado. Outras reuniões foram feitas com os pais ou responsáveis. Nessas reuniões, além da apresentação do trabalho, foi solicitada a autorização para a realização do estudo e o preenchimento do questionário socioeconômico.

As reuniões foram feitas no início do semestre letivo de 2002, aproveitando a reunião dos pais e responsáveis com a escola. Nesse período, a participação dos pais é significativa, inclusive porque o transporte dos pais e responsáveis até a escola é gratuito. $\mathrm{O}$ contato com os pais foi feito através de cartas ou bilhetes enviados pela direção da escola. Nesse contato, além do motivo principal da reunião (questões específicas da escola), os pais foram informados acerca da participação do pesquisador. Foram feitas oito reuniões, uma em cada comunidade pesquisada. A participação dos pais e responsáveis foi grande, garantindo o número de 476 participantes. O pesquisador principal realizou as entrevistas para preenchimento do questionário.

A classificação da ocupação do pai e da mãe foi feita em duas etapas. $\mathrm{Na}$ primeira etapa, foi utilizada a Classificação Nacional de Atividades Econômicas (CNAE) do Instituto Brasileiro de Geografia e Estatística (IBGE) (29). Numa segunda etapa, com base no referencial teórico apresentado por Graziano-Silva (30), as ocupações dos pais foram dicotomizadas em ocupações rurais agrícolas e ocupações rurais não-agrícolas. Os conceitos de renda familiar e de escolaridade do pai e da mãe do escolar foram os mesmos utilizados pelo Ministério da Saúde (31). O consumo de água foi dicotomizado entre a utilização do Serviço Autônomo de Água e Esgoto (SAAE) (água clorada) ou de outra fonte (mina, cisterna, poço artesiano particular, rio, ribeirão e açude, entre outros).

Para garantir a confiabilidade dos dados sobre cárie dentária, realizou-se uma padronização de diagnóstico com um grupo de 20 crianças. $\mathrm{O}$ pesquisador, respeitando todas as normas de controle de infecção, examinou, em dois momentos, as mesmas crianças, utilizando os mesmos critérios diagnósticos. Os exames foram realizados com intervalo de 1 semana. O teste kappa mostrou concordância ótima entre os diagnósticos realizados em cada um dos exames (kappa > 0,90) (32). Além disso, para testar a metodologia proposta neste trabalho, foram examinados, como parte de um estudo piloto, todos os escolares em uma das comunidades rurais. Toda a metodologia mostrou-se adequada. Posteriormente, as crianças dessa comunidade foram reexaminadas no estudo principal.

O estudo principal para a cárie dentária baseou-se na metodologia preconizada pela Organização Mundial da Saúde (OMS) (32) para levantamentos epidemiológicos em saúde bucal. As crianças foram examinadas na escola, sob luz natural, por um único examinador. Antes do exame, os alunos ganhavam uma escova de dentes e realizavam escovação dentária orientada, visando a melhorar as condições do exame. Todas as informações foram registradas em formulário específico por uma anotadora treinada para a função.

A cada 10 crianças examinadas, um exame foi realizado em duplicata. $\mathrm{O}$ 
examinador não era informado desse exame em duplicata para não interferir no resultado da reprodutibilidade. $\mathrm{O}$ teste kappa revelou um nível ótimo de concordância entre os diagnósticos dos exames realizados em duplicata (kappa $>0,90)$.

A severidade de cárie, para cada indivíduo, foi mensurada através do número de dentes permanentes cariados, perdidos e restaurados (CPOD) e do número de dentes decíduos cariados, com extração indicada e obturados (ceod). Posteriormente, o cálculo do CPOD e do ceod foi realizado para o grupo como um todo. Além disso, foi mensurada a prevalência de cárie nas dentições permanente e decídua e em ambas as dentições (32).

\section{Análise estatística}

Um banco de dados foi montado no programa Statistical Package for the Social Sciences (SPSS) versão 11 (33). A análise estatística descritiva baseou-se em cálculo de proporções, medidas de tendência central e de variabilidade para as variáveis prevalência de cárie dentária nas dentições permanente e decídua, severidade de cárie dentária (índices CPOD e ceod) e variáveis socioeconômicas. Para a avaliação da normalidade das variáveis foi utilizado o teste de Kolmogorov-Smirnov. Toda a análise estatística dos dados (univariada e multivariada) foi realizada considerando o agrupamento de idades estatisticamente semelhantes em relação à experiência de cárie $(P>$ $0,05)$ em quatro grupos etários: 4 a 6 anos; 7 e 8 anos; 9 e 10 anos; e 11 a 15 anos. A estratificação da análise estatística pela idade é utilizada por diversos estudos epidemiológicos sobre cárie dentária, considerando a forte influência da faixa etária sobre a doença $(1,3,15,17,20,21)$.

Uma análise univariada foi realizada para testar a influência das variáveis socioeconômicas (variáveis independentes) na prevalência de cárie dentária (variável dependente). A variável dependente foi dicotomizada entre ausência e presença da doença cárie nas dentições decídua e perma- nente e em ambas as dentições (experiência total de cárie). $\mathrm{O}$ teste do quiquadrado $\left(\chi^{2}\right)$ e o teste exato de Fisher foram utilizados nesta etapa, considerando o nível de significância estatística de 0,05 (33). A variável renda foi dicotomizada pelo valor mediano e as variáveis escolaridade do pai e da mãe foram dicotomizadas em até 3 anos de escolaridade formal (ensino fundamental incompleto) e mais do que 3 anos de escolaridade, para melhor ajuste do modelo logístico. Foram construídos modelos de regressão logística múltipla para as variáveis dependentes que apresentaram mais de uma covariável estatisticamente associada $(P<0,25)$. A opção por utilizar um nível de significância estatística mais alto é recomendada pelos autores que estudam regressão logística. Tais autores discutem que o uso de um nível de significância mais tradicional (como 0,05) freqüentemente não identifica variáveis importantes para o modelo logístico $(34,35)$.

Para avaliar a multicolinearidade (presença de covariáveis fortemente correlacionadas e que poderiam interferir no ajuste do modelo logístico) foram calculados os coeficientes de correlação de Spearman para as covariáveis quantitativas. Foram calculadas a razão de chances (odds ratio, OR) e o intervalo de confiança de 95\% (IC95\%) da OR para cada uma das covariáveis. O teste de Hosmer e Lemeshow foi utilizado para avaliar a adequação do modelo logístico final $(34,35)$.

\section{RESULTADOS}

Fizeram parte do estudo 476 (94,63\%) alunos matriculados no ensino público do meio rural de Itaúna. A perda de participantes não foi superior a $20 \%$ em nenhuma das comunidades pesquisadas.

\section{Caracterização socioeconômica}

A idade dos participantes variou de 4 a 15 anos, com média igual a 7,64 anos $( \pm 2,19) ; 91,8 \%$ dos alunos tinham até 10 anos de idade; havia 262 meni- nos (55\%) e 214 meninas (45\%). Ocupações rurais não-agrícolas foram referidas por $50,7 \%$ dos pais. Entre as mães, 93,9\% tinham ocupações rurais não-agrícolas, sendo 406 donas de casa. A escolaridade média dos pais, em anos de estudo formal, foi baixa, sendo igual a 3,62 $( \pm 2,31)$ entre os homens e 4,14 $( \pm 2,41)$ entre as mulheres. Entre os pais, 80,7\% tinham até 4 anos de estudo formal. Entre as mães, essa proporção foi de $76,0 \%$.

A renda média mensal da população era de 313,84 reais $( \pm 190,26)$, sendo que $78,2 \%$ das famílias recebiam até 2 salários-mínimos (na época, o saláriomínimo era de $\mathrm{R} \$ 180$, ou aproximadamente 76 dólares). Apenas 28,3\% das famílias utilizavam a água tratada com cloro fornecida pelo SAAE local.

\section{Cárie dentária}

O total de crianças com experiência de cárie foi igual a 391 escolares. Ou seja, a prevalência de cárie para esses indivíduos, considerando ambas as dentições, totalizou 82,14\%. Houve, ainda, 85 (17,86\%) escolares que nunca haviam apresentado a doença cárie dentária em nenhuma das dentições.

Quatrocentos e dois escolares apresentavam pelo menos um dente permanente. A prevalência de cárie na dentição permanente desse grupo foi de $37,3 \%$, e o índice CPOD médio foi igual a $0,94( \pm 1,55)$. O índice médio equivale à soma da média para cada um dos componentes do CPOD: 0,34 $( \pm 0,78)$ dentes permanentes cariados, $0,03( \pm 0,23)$ dentes perdidos e 0,57 ( \pm $1,23)$ elementos restaurados.

Das crianças com dentes permanentes, 62,7\% apresentaram CPOD igual a zero. $\mathrm{O}$ valor médio do CPOD excluindo essas crianças foi de 2,51 $( \pm 1,57)$. As médias dos componentes do índice (dentes permanentes cariados, dentes permanentes perdidos e dentes permanentes restaurados) foram iguais a $0,91( \pm 1,05), 0,07( \pm 0,37)$ e $1,53( \pm 1,61)$, respectivamente.

Os dentes permanentes mais acometidos pela cárie foram os primeiros molares, totalizando $89,42 \%$ da experiência da doença. Desses dentes, 72 
necessitavam de restauração em uma superfície, 23 necessitavam de restaurações em duas superfícies e 18 necessitavam de tratamento pulpar mais restauração. A extração era indicada em apenas cinco dentes. Um primeiro molar necessitava de restauração tipo coroa total.

O índice ceod médio para toda a população que apresentava dentes decíduos $(\mathrm{n}=435)$ foi igual a $4,00( \pm 3,46)$. As médias de cada componente do índice (dentes decíduos cariados, dentes decíduos com extração indicada e dentes decíduos restaurados) foram, respectivamente, 2,21 $( \pm 2,75), 0,26$ $( \pm 0,26)$ e $1,53( \pm 2,02)$.

Os índices CPOD e ceod seguiram uma tendência oposta após os 6 anos de idade, ou seja: com o aumento da idade, observou-se uma redução no índice ceod, enquanto que o CPOD tendeu a aumentar com a idade (figuras 1 e 2). $\mathrm{O}$ índice ceod médio, excluindo as 82 crianças livres de cárie na dentição decídua, foi igual a 4,93 ( $\pm 3,18)$. As médias dos componentes do índice (dentes decíduos cariados, dentes decíduos com extração indicada e dentes decíduos restaurados) foram, respectivamente, $2,73( \pm 2,82), 0,32( \pm 0,90)$ e $1,88( \pm 2,09)$.

Os dentes decíduos mais acometidos pela cárie foram os primeiros e segundos molares, representando $80,31 \%$ de toda a experiência da doença. Desse total, 135 molares decíduos necessitaram de restauração em uma superfície, 424 de restauração em duas superfícies, 139 necessitam de tratamento pulpar e restauração e 78 precisavam ser extraídos devido à cárie dentária.

\section{Análise univariada}

As tabelas de 1 a 3 apresentam a prevalência de cárie em termos do sexo da criança, da ocupação dos pais e da escolaridade dos pais para cada uma das faixas etárias consideradas. A tabela 4 apresenta a prevalência de cárie em termos da renda familiar e da origem da água consumida para cada uma das faixas etárias.

Apenas a escolaridade da mãe, nas crianças com 7 e 8 anos de idade, es-

\section{FIGURA 1. Distribuição do índice ceod em escolares do meio rural de Itaúna (MG), Brasil, 2002}

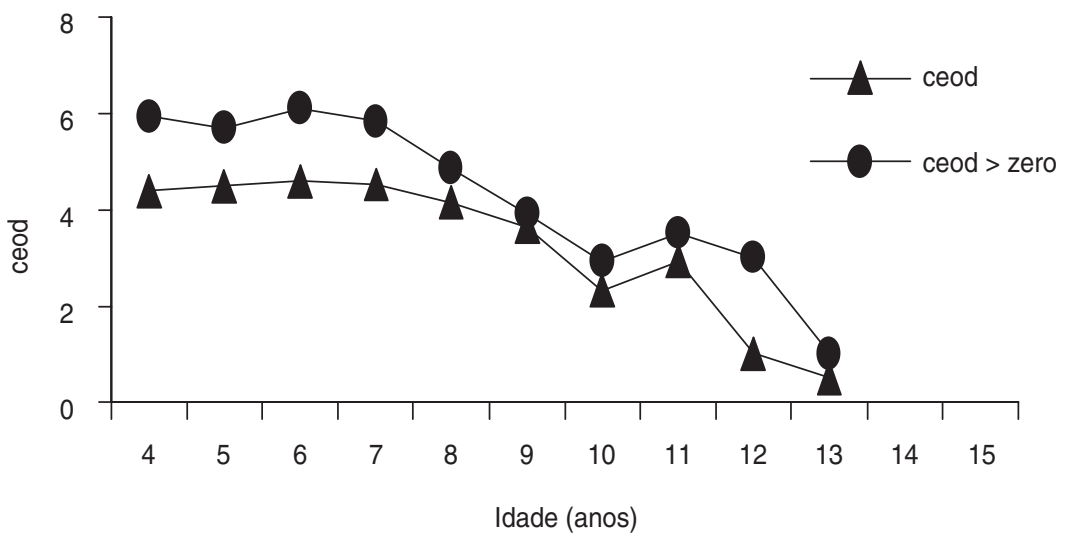

${ }^{a}$ ceod = Número de dentes decíduos cariados, com extração indicada e obturados. A curva de ceod > zero indica o ceod médio excluindo os escolares livres de cárie. teve estatisticamente associada com a prevalência de cárie na dentição permanente (tabela 3). A única variável que esteve estatisticamente associada com a experiência de cárie na dentição decídua foi a renda familiar (tabela 4) entre as crianças de 7 a 8 anos de idade. Observou-se, ainda, que a covariável renda esteve associada com a experiência total de cárie na faixa etária de 7 a 8 anos.

\section{Análise multivariada}

A maior correlação identificada na avaliação da multicolinearidade foi entre escolaridade do pai e da mãe $(0,324)$. Apesar dessa correlação ser estatisticamente significativa $(P=0,000)$, a mesma pode ser considerada fraca. Assim, todas as covariáveis associadas com a variável dependente, em um nível de significância estatístico de $P<$

\section{FIGURA 2. Distribuição do índice CPOD médio em escolares do meio rural de Itaúna (MG), Brasil, 2002 ${ }^{\mathrm{a}}$}

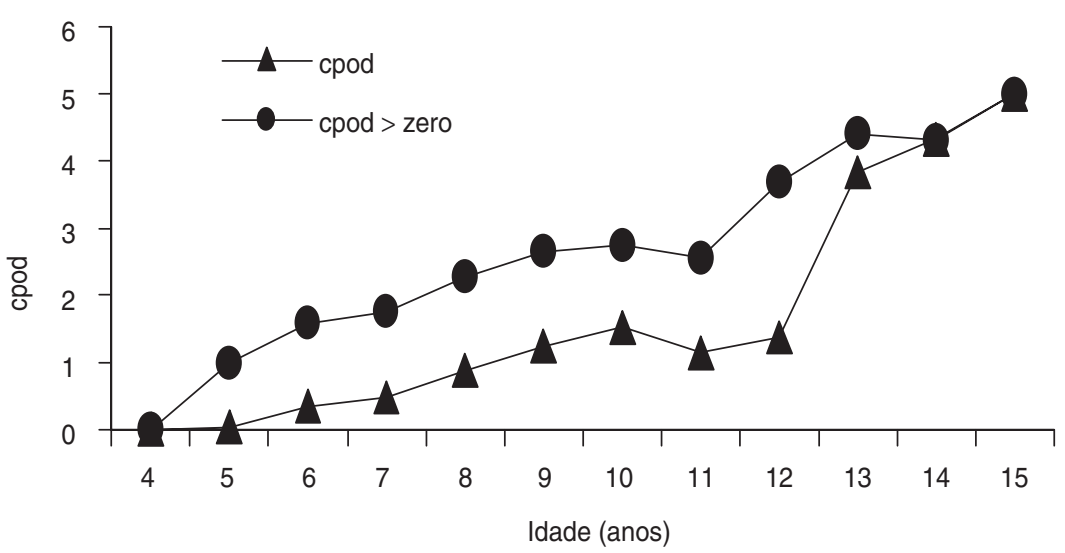

a $\mathrm{CPOD}=$ Número de dentes permanentes cariados, perdidos e restaurados. $\mathrm{A}$ curva de $\mathrm{CPOD}>$ zero indica - CPOD médio excluindo os escolares livres de cárie. 
TABELA 1. Análise univariada da associação entre o sexo e a prevalência de cárie, meio rural de ltaúna (MG), Brasil, 2002ª

\begin{tabular}{|c|c|c|c|c|c|c|c|c|c|c|}
\hline \multirow[b]{2}{*}{ Idade (anos) } & \multirow[b]{2}{*}{ Sexo } & \multicolumn{3}{|c|}{$\mathrm{CPOD}^{\mathrm{b}}$ (No.) } & \multicolumn{3}{|c|}{$\operatorname{ceod}^{\mathrm{C}}$ (No.) } & \multicolumn{3}{|c|}{$\begin{array}{l}\text { Experiência total } \\
\text { de cáried }^{d} \text { (No.) }\end{array}$} \\
\hline & & Ausente & Presente & $P$ & Ausente & Presente & $P$ & Ausente & Presente & $P$ \\
\hline 4 a 6 & Feminino & 32 & 7 & 0,56 & 19 & 51 & 0,19 & 17 & 53 & 0,37 \\
\hline \multirow[t]{2}{*}{7 a 8} & Masculino & 55 & 23 & & 16 & 63 & & 15 & 64 & \\
\hline & Feminino & 45 & 25 & 0,42 & 12 & 58 & 0,63 & 11 & 59 & 0,60 \\
\hline 9 a 10 & Masculino & 32 & 34 & & 7 & 52 & & 6 & 60 & \\
\hline 11 a 15 & Feminino & 4 & 5 & 1,00 & 1 & 4 & 1,00 & 2 & 7 & 1,00 \\
\hline
\end{tabular}

${ }^{\mathrm{a}} n=476$ escolares.

${ }^{b} \mathrm{CPOD}=$ Número de dentes permanentes cariados, perdidos e restaurados

${ }^{c}$ ceod $=$ Número de dentes decíduos cariados, com extração indicada e obturados.

d Experiência de cárie em ambas as dentições.

TABELA 2. Análise univariada da associação entre a ocupação do pai e da mãe e a prevalência de cárie, meio rural de ltaúna (MG), Brasil, 2002ª

\begin{tabular}{|c|c|c|c|c|c|c|c|c|c|c|}
\hline \multirow[b]{2}{*}{ Idade (anos) } & \multirow[b]{2}{*}{ Ocupação } & \multicolumn{3}{|c|}{$\mathrm{CPOD}^{\mathrm{b}}$ (No.) } & \multicolumn{3}{|c|}{$\operatorname{ceod}^{c}$ (No.) } & \multicolumn{3}{|c|}{$\begin{array}{l}\text { Experiência total } \\
\text { de cáried (No.) }^{\text {d }}\end{array}$} \\
\hline & & Ausente & Presente & $P$ & Ausente & Presente & $P$ & Ausente & Presente & $P$ \\
\hline \multirow[t]{2}{*}{4 a 6} & Rural agrícola & 26 & 6 & & 18 & 48 & & 18 & 48 & \\
\hline & Rural não-agrícola & 42 & 5 & 0,34 & 17 & 67 & 0,31 & 15 & 69 & 0,17 \\
\hline \multirow[t]{2}{*}{7 a 8} & Rural agrícola & 51 & 24 & & 15 & 61 & & 13 & 63 & \\
\hline & Rural não-agrícola & 45 & 23 & 0,82 & 10 & 58 & 0,43 & 10 & 58 & 0,70 \\
\hline \multirow[t]{3}{*}{11 a 15} & Rural agrícola & 9 & 12 & & 2 & 8 & & 4 & 17 & \\
\hline & Rural não-agrícola & 10 & 12 & 0,92 & 3 & 4 & 0,59 & 6 & 12 & 0,47 \\
\hline & Mãe & & & & & & & & & \\
\hline \multirow[t]{2}{*}{4 a 6} & Rural agrícola & 5 & 1 & & 4 & 8 & & 4 & 8 & \\
\hline & Rural não-agrícola & 65 & 10 & 1,00 & 31 & 109 & 0,47 & 29 & 111 & 0,30 \\
\hline 7 a 8 & Rural agrícola & 5 & 2 & & 1 & 6 & & 1 & 6 & \\
\hline 11 a 15 & Rural não-agrícola & 15 & 22 & 0,42 & 5 & 10 & 1,00 & 10 & 27 & 1,00 \\
\hline
\end{tabular}

a $n=476$ escolares.

${ }^{\mathrm{b}} \mathrm{CPOD}=$ Número de dentes permanentes cariados, perdidos e restaurados.

${ }^{c}$ ceod= Número de dentes decíduos cariados, com extração indicada e obturados.

d Experiência de cárie em ambas as dentições.

0,25, foram incluídas na construção dos modelos logísticos $(34,35)$.

A escolaridade da mãe manteve sua associação estatística com a prevalência de cárie na dentição permanente. Pode-se considerar que crianças com idade entre 7 e 8 anos cujas mães tinham escolaridade superior a 3 anos tinham 2,813 vezes mais chance
(IC95\%: 1,221 a 6,480) de apresentar cárie na dentição permanente quando comparadas com crianças cujas mães apresentavam escolaridade mais baixa. A renda familiar manteve-se associada com a prevalência de cárie dentária na dentição decídua, bem como na prevalência total de cárie na faixa etária de 7 e 8 anos. Em relação à dentição decídua, observou-se que escolares com renda familiar mensal superior a $\mathrm{R} \$ 280$ tinham 2,602 vezes mais chance (IC95\%: 1,004 a 6,745) de apresentar a doença cárie do que aqueles com renda mensal inferior a $\mathrm{R} \$ 280$. Em relação à prevalência total de cárie, identificou-se que crianças cuja renda familiar mensal era superior a $\mathrm{R} \$ 280$ 
TABELA 3. Análise univariada da associação entre a escolaridade do pai e da mãe e a prevalência de cárie, meio rural de ltaúna (MG), Brasil, $2002^{\mathrm{a}}$

\begin{tabular}{|c|c|c|c|c|c|c|c|c|c|c|}
\hline \multirow[b]{2}{*}{ Idade (anos) } & \multirow{2}{*}{$\begin{array}{l}\text { Escolaridade } \\
\text { (anos) }\end{array}$} & \multicolumn{3}{|c|}{$\mathrm{CPOD}^{\mathrm{b}}$ (No.) } & \multicolumn{3}{|c|}{$\operatorname{ceod}^{c}$ (No.) } & \multicolumn{3}{|c|}{$\begin{array}{c}\text { Experiência total } \\
\text { de cárie }^{d}(\text { No.) }\end{array}$} \\
\hline & & Ausente & Presente & $P$ & Ausente & Presente & $P$ & Ausente & Presente & $P$ \\
\hline \multirow[t]{2}{*}{4 a 6} & Até 3 & 23 & 3 & & 12 & 34 & & 12 & 34 & \\
\hline & Mais de 3 & 44 & 8 & 0,74 & 23 & 81 & 0,60 & 21 & 83 & 0,42 \\
\hline 7 a 8 & Até 3 & 41 & 13 & & 12 & 43 & & 12 & 43 & \\
\hline \multirow[t]{3}{*}{11 a 15} & Até 3 & 10 & 15 & & 3 & 8 & & 5 & 20 & \\
\hline & Mais de 3 & 6 & 7 & 0,72 & 1 & 4 & 1,00 & 4 & 9 & 0,69 \\
\hline & Mãe & & & & & & & & & \\
\hline \multirow[t]{2}{*}{4 a 6} & Até 3 & 19 & 2 & & 9 & 23 & & 9 & 23 & \\
\hline & Mais de 3 & 49 & 9 & 0,72 & 26 & 93 & 0,46 & 24 & 95 & 0,33 \\
\hline 7 a 8 & Até 3 & 37 & 9 & & 10 & 37 & & 10 & 37 & \\
\hline 11 a 15 & Mais de 3 & 10 & 12 & 0,62 & 2 & 7 & 0,60 & 6 & 16 & 1,00 \\
\hline
\end{tabular}

a $n=476$ escolares.

${ }^{\mathrm{b}} \mathrm{CPOD}=$ Número de dentes permanentes cariados, perdidos e restaurados.

${ }^{c}$ ceod $=$ Número de dentes decíduos cariados, com extração indicada e obturados.

d Experiência de cáries em ambas as dentições.

apresentaram 2,854 vezes mais chance (IC95\%: 1,044 a 7,799) de terem cárie dentária do que as crianças com renda familiar mensal inferior a R\$ 280.

\section{DISCUSSÃO}

O presente trabalho avaliou a cárie dentária, maior problema de saúde bucal coletiva no Brasil, nos escolares do meio rural de Itaúna. Levando-se em consideração que todas as crianças do meio rural estão matriculadas no ensino fundamental, este estudo apresenta dados de quase a totalidade das crianças em idade escolar no meio rural da região. Esse ponto é considerado importante, uma vez que a maioria dos trabalhos sobre cárie dentária realizados no Brasil utilizaram técnicas de amostragem por conveniência ou não relataram a técnica de amostragem (7-9, 11, 12, 14, 15). Poucos estudos utilizaram amostragem probabilística $(13,16)$ ou analisaram o universo de crianças $(5,10,15,17)$. Além disso, poucos estudos $(7,8)$ avaliaram a den- tição decídua no meio rural brasileiro, que tem sido considerada um motivo de preocupação em relação à epidemiologia da cárie no Brasil $(2,36)$. Os dados apresentados pelo presente estudo podem ser considerados confiáveis, considerando-se a concordância intra-examinador mensurada.

Além disso, a grande maioria dos estudos epidemiológicos sobre cárie dentária no Brasil mostrou grandes necessidades acumuladas em relação à doença, ou seja, descreveu populações sem assistência odontológica sistemática. Em Itaúna, há um programa de assistência odontológica organizado pelo SUS local que envolve o atendimento odontológico clínico e atividades coletivas educativas. Dessa forma, avaliar a doença cárie em populações rurais que recebem atenção odontológica com uma certa regularidade é um aspecto pouco relatado na literatura nacional e internacional e, por conseqüência, também pode permitir um avanço no entendimento dessa doença.

Em nível nacional, as discussões mais recentes sobre o meio rural reve- lam características heterogêneas em relação, por exemplo, à inserção produtiva e à renda dos moradores de diferentes zonas rurais $(30,37-39)$. Os escolares do meio rural de Itaúna, no entanto, vivem em um meio com características socioeconômicas bastante homogêneas. A única variável avaliada que confirmou a heterogeneidade entre as diferentes comunidades rurais de Itaúna foi a ocupação do pai. A maioria dos escolares estudados era proveniente de famílias de renda muito baixa, cujos pais tinham baixo nível de escolaridade; apenas a minoria tinha acesso à água tratada. Esses indicadores sociais desfavoráveis refletem a distribuição de renda desigual do Brasil, onde mais de 57 milhões de indivíduos vivem abaixo da linha da pobreza (40).

Em relação à prevalência de cárie nos dentes permanentes, observa-se que os estudos de Andrioni et al. (7), Marcos et al. (9) e Makowiecky e Silva (11) identificaram uma proporção bem superior de indivíduos com cárie dentária em dentes permanentes do que os 
TABELA 4. Análise univariada da associação entre a renda familiar mensal e a origem da água consumida com prevalência de cárie, meio rural de Itaúna (MG), Brasil, 2002 ${ }^{\mathrm{a}}$

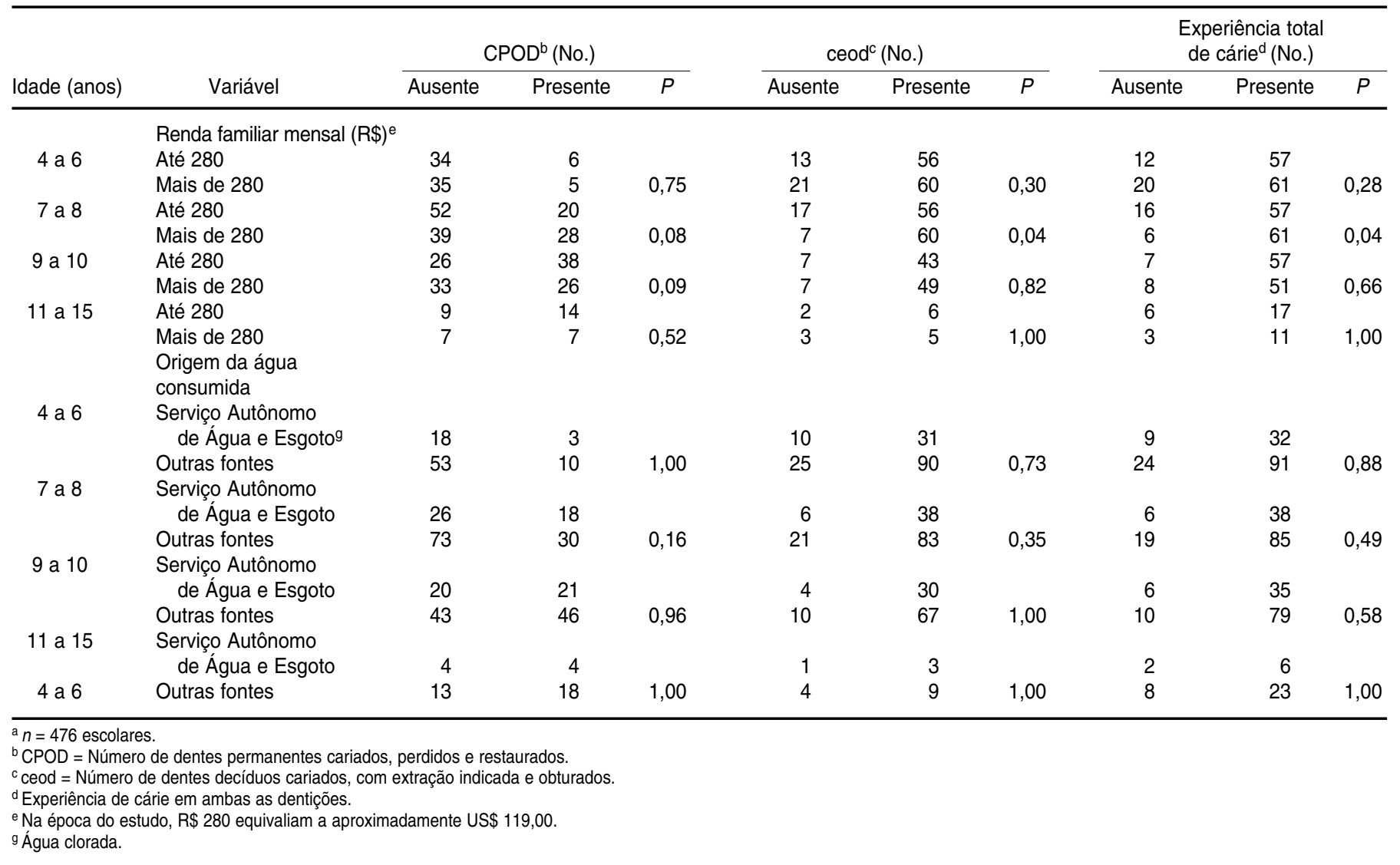

achados do presente trabalho. Essa diferença pode estar associada a mudanças nos critérios de diagnóstico da cárie dentária, implementadas a partir da década de 1990 (41). Além disso, o consumo de dentifrícios fluoretados, largamente utilizados pela população brasileira desde o final da década de 1980, é um dos principais responsáveis pelo declínio da cárie (42), e pode ter sido um importante fator para explicar a menor prevalência da doença observada no presente estudo. Mesmo assim, comparando-se os achados de Itaúna com estudos mais recentes, verifica-se que a prevalência da cárie dentária na dentição permanente (37,3\%) também pode ser considerada bastante inferior àquela encontrada por Dini e Silva (13) em Araraquara, Estado de São Paulo (66,8\%); por Furtado et al. (15) em Capão Alto, Estado de Santa Catarina (75,0\%); por Sampaio et al. (6), que estudaram o meio rural da Paraíba $(81,96 \%)$; e por Traebert et al. (17), no Estado de Santa Catarina $(62,1$ e $63,6 \%$, para as duas comunidades pesquisadas).

Em relação aos valores do CPOD médio, verifica-se que em Itaúna a severidade de cárie dentária foi menor do que aquela relatada por todos os estudos brasileiros em populações rurais, exceto por Araújo et al. (14). Quando se comparam os dados do presente trabalho com os dados nacionais do Ministério da Saúde de 1996, verifica-se que o meio rural de Itaúna, mais uma vez, apresenta menor severidade de cárie dentária na dentição permanente, exceto nas crianças de 6 anos (36). No entanto, a população rural de Itaúna apresenta uma severidade mais alta de cárie dentária do que a de Belo Horizonte, capital de Minas Gerais (36). É difícil apontar com muita certeza quais foram os motivos de tais diferenças, uma vez que os outros estudos brasileiros foram apenas descritivos. Fatores sociais e biológicos podem ser diferentes nessas localidades rurais, contribuindo para a maior ou menor prevalência da doença. A idade, que é mais baixa no presente estudo em relação a alguns outros trabalhos, também pode ter contribuído para essas diferenças. Outro aspecto que poderia explicar esse resultado, pelo menos em parte, é a presença do serviço de saúde bucal. Em todos os outros estudos sobre saúde bucal de populações rurais, observa-se a ausência quase completa de assistência odontológica. É possível que as medidas adotadas pelo SUS tenham melhorado as condições de 
saúde bucal na dentição permanente desse grupo. No entanto, as hipóteses levantadas para explicar as diferenças identificadas merecem ser avaliadas posteriormente através de estudos apropriados para tais objetivos.

É importante ressaltar que nem os dados de CPOD, nem os de ceod, apresentam distribuição gaussiana, conforme revelou o teste de KolmogorovSmirnov. Assim, a média deve ser interpretada com cautela, não sendo um indicador muito útil para o planejamento das ações do serviço de saúde. A forma mais interessante de interpretar esses dados é através de proporções. Por exemplo, 91,5\% das crianças apresentavam até três dentes permanentes com experiência de cárie (368/402). Da mesma forma, o percentual de crianças livres de cárie na dentição decídua, excluindo os 41 escolares que não apresentavam dentes decíduos, foi igual a 18,85\% (82/435).

A concentração do CPOD em primeiros molares confirma outros achados descritos na literatura $(8,10,43)$. $\mathrm{O}$ tratamento restaurador nesses elementos dentários é geralmente de baixa complexidade e de fácil resolução pelo SUS local. É preciso considerar, porém, que essa população, embora jovem, já necessita de tratamento endodôntico. Para esses casos, o poder público municipal deveria organizar tal atendimento, de forma a evitar perdas dentárias precoces.

Foi observado um aumento no índice CPOD (incluindo ou não os indivíduos livres de cárie) com o aumento da idade, com exceção da faixa acima de 10 anos, na qual ocorreu uma diminuição do CPOD (figura 2). Os dados para as crianças acima de 10 anos, no entanto, devem ser interpretados com cautela, pois os moradores do meio rural cursam as quatro últimas séries do ensino fundamental no meio urbano de Itaúna. Assim, as crianças com idade superior a 10 anos não constituem uma amostra representativa dos moradores do meio rural de Itaúna.

Em relação ao ceod médio no meio rural de Itaúna, observa-se que esse valor é superior àquele apresentado por Andrioni et al. (7) e inferior ao de
Bijella e Bijella (8), os dois únicos estudos brasileiros que avaliaram a dentição decídua no meio rural. Em relação aos dados de 1996 referentes ao Brasil e a Belo Horizonte, observa-se que as crianças do meio rural de Itaúna apresentaram maior severidade de cárie dentária na dentição decídua (36).

Internacionalmente, os estudos realizados no continente africano (44-46) mostram uma menor severidade de cárie na dentição decídua comparandose com as crianças itaunenses. Vários fatores podem explicar essa diferença, dentre eles o consumo de sacarose. Embora o consumo de sacarose não tenha sido avaliado no presente estudo, sabe-se que no Brasil o consumo de sacarose é bem maior do que na África. Alguns países asiáticos, por outro lado, apresentam maior severidade de cárie na dentição decídua do que a observada no presente trabalho (47-49).

A diminuição do ceod com o aumento da idade pode ser explicado pela esfoliação ou perda fisiológica dos dentes decíduos que ocorre ao longo da vida. Com a diminuição dos dentes decíduos na cavidade bucal, elementos dentários que poderiam apresentar experiência de cárie são perdidos. Assim, a severidade da doença diminui (figura 1). Esses achados confirmam outros relatos na literatura $(7,8,44,45,49)$.

A OMS colocou como uma de suas metas de saúde bucal para o ano de 2000 que $50 \%$ das crianças com idade entre 5 e 6 anos estivessem livres de cárie. Para o ano de 2010, a meta é de $90 \%$ das crianças livres de cárie (50). No entanto, no meio rural de Itaúna, apenas $21,37 \%$ das crianças nessa faixa etária apresentaram tal condição. Tais achados reforçam a preocupação em relação à dentição decídua no Brasil (2, 20, 51).

Os dados do presente estudo, principalmente na dentição decídua, mostram que é necessária e urgente a implementação de estratégias coletivas em saúde bucal em uma faixa etária mais precoce. $\mathrm{O}$ fornecimento, pelo SAAE, de água fluoretada, método considerado importante para o controle da cárie, beneficiaria menos de um terço das famílias estudadas. Portanto, a utilização isolada desse método não teria um impacto forte sobre a saúde bucal. O SUS local deve, com a participação da população rural, e de maneira intersetorial (52), discutir quais seriam as estratégias mais adequadas para lidar com as questões epidemiológicas levantadas por este trabalho.

Além disso, seria aconselhável instituir um processo de vigilância das condições de saúde bucal nessa população, principalmente quando se considera que a experiência passada de cárie é considerada uma importante medida para se avaliar o risco de cárie no futuro (53). Assim, os resultados favoráveis acerca da experiência atual de cárie na dentição permanente no meio rural poderiam ser modificados no futuro.

Um dos aspectos analisados neste estudo foi a determinação socioeconômica da cárie dentária. Sabe-se que essa área tem sido analisada por diversos autores nacionais e internacionais (3, 19-27). Assim como em outros estudos $(7,11)$, o gênero das crianças não se mostrou estatisticamente associado com a experiência de cárie. $\mathrm{O}$ acesso à água tratada poderia ser um indicador de maior consciência sanitária, bem como de melhores condições socioeconômicas (51), e, conseqüentemente, poderia estar associado a melhores condições de saúde. No entanto, essa hipótese não foi confirmada.

A ocupação dos pais foi avaliada considerando as mudanças sociais identificadas no meio rural brasileiro na última década (30). Supunha-se que indivíduos que apresentassem ocupações rurais agrícolas pudessem apresentar hábitos alimentares mais tradicionais e, portanto, menor risco de cárie. Por outro lado, o contato dos pais que apresentavam ocupações rurais não-agrícolas com atividades de estilo mais urbano poderia levá-los a um maior contato com hábitos alimentares ricos em sacarose. No entanto, não houve associação entre a doença e essas variáveis.

Em relação à escolaridade dos pais e à renda, o primeiro aspecto que chama a atenção é a homogeneidade da po- 
pulação rural estudada. Como já discutido anteriormente, os indicadores sociais são muito desfavoráveis para a grande maioria da população, ou seja, esta população compartilha a mesma condição de pobreza. Assim, para a maioria das faixas etárias, não foram identificadas associações estatisticamente significativas entre cárie dentária e as variáveis escolaridade dos pais e renda familiar mensal. Em relação a esse achado, pode-se recorrer às discussões de Rose (54). Segundo aquele autor, quando as populações estão expostas de forma homogênea a um certo fator de risco, torna-se extremamente difícil identificar, através dos métodos epidemiológicos analíticos, a relação entre esse fator de exposição e a doença pesquisada.

Entretanto, a regressão logística revelou, na faixa etária de 7 a 8 anos, associações estatisticamente significativas entre algumas covariáveis e os indicadores de cárie. A renda familiar mais alta esteve estatisticamente associada com maior chance de cárie na dentição decídua e em ambas as dentições. A escolaridade mais alta da mãe esteve associada com maior prevalência de cárie na dentição permanente. Esses achados vão de encontro a diversos estudos de acordo com os quais os grupos com condições sociais e econômicas menos favoráveis apresentam maior experiência da doença (3, 19-27). Tais estudos, entretanto, enfocaram populações urbanas e populações que apresentavam grandes diferenças sociais e econômicas. Para populações com privações econômicas importantes, como é o caso do meio rural de Itaúna, um pouco mais de renda e educação poderia significar mais acesso a alimentos refinados (55, 56) e, portanto, maior prevalência de cárie. O estudo de Al-Hosani e RuggGunn (47) apresentou a mesma associação socioeconômica identificada no presente trabalho. Essa confirmação, apesar de rara na literatura, mostra-se consistente, pois existem características metodológicas bastante semelhantes entre os trabalhos: ambos foram desenvolvidos no meio rural, avaliando a dentição decídua, com coleta de informações e análise estatística muito semelhantes.

A associação direta entre escolaridade da mãe e cárie dentária pode ser conseqüência das características específicas do meio rural estudado. Em Itaúna, como comentado anteriormente, apenas as quatro primeiras séries do ensino fundamental são cursadas no meio rural. As séries subseqüentes são cursadas no meio urbano. Podese especular, então, que as mães com mais de 3 anos de ensino formal tiveram um contato maior com o meio urbano. Dessa forma, é possível que essas mães com maior escolaridade tenham uma maior influência urbana na sua alimentação e, por conseqüência, consumam mais sacarose. Estudos realizados em outros países mostram que o processo de urbanização está diretamente associado com o aparecimento da doença cárie, devido à ocidentalização dos hábitos alimentares $(45,57,58)$.

Finalmente, estudos com maior poder analítico merecem ser realizados no meio rural brasileiro para que se possa entender a complexa interação entre determinantes socioeconômicos e a doença cárie. As pesquisas qualitativas também podem ajudar na interpretação dos dados identificados através dessa metodologia transversal, para que se possam avaliar os processos simbólicos na determinação social das doenças (59) e, mais especificamente, da doença cárie.

Agradecimentos. O estudo foi desenvolvido com verbas do Conselho Nacional de Desenvolvimento Científico e Tecnológico (CNPq) e da Fundação de Amparo à Pesquisa do Estado de Minas Gerais (FAPEMIG). Os autores agradecem à Prefeitura de Itaúna pelo apoio na condução da pesquisa.

\section{REFERÊNCIAS}

1. Brasil, Ministério da Saúde. Levantamento epidemiológico em saúde bucal. $1^{a}$ etapa - cárie dental. Brasília: Ministério da Saúde; 1996.

2. Pinto VG. Epidemiologia das doenças bucais no Brasil. Em: Kriger L, org. ABOPREV: promoção de saúde bucal. São Paulo: Artes Médicas; 1997. Pp. 27-41.

3. Brasil, Ministério da Saúde. Levantamento epidemiológico em saúde bucal: Brasil, zona urbana, 1986. Brasília: Centro de Documentação do Ministério da Saúde; 1988.

4. Instituto Brasileiro de Geografia e Estatística. Censo demográfico 2000 - resultado do universo. Disponível em: http://www.ibge.gov. $\mathrm{br} /$ censo/default.php. Acessado em 21 de setembro de 2004.

5. Abreu MHNG. Dimensões epidemiológicas da saúde bucal da população rural de Itaúna, Minas Gerais, 2002 [tese]. Belo Horizonte: Es- cola de Veterinária da Universidade Federal de Minas Gerais; 2004.

6. Sampaio FC, Nazmul Hossain ANM, Von de Feher FR, Arneberg P. Dental caries and sugar intake of children from rural areas with different water fluoride levels in Paraíba, Brazil. Community Dent Oral Epidemiol. 2001;28(4): 307-13.

7. Andrioni JN, Benfatti SV, Russo MC, Toledo OA. Prevalência de gengivite e de cárie dental. Rev Bras Odontol. 1969;26(155):5-10.

8. Bijella MFTB, Bijella VT. Prevalência da cárie dental em escolares da zona rural de 12 municípios da região de Bauru. Rev Bras Odontol. 1970;27(163):133-8.

9. Marcos B, Araújo MS, Moraes MM, Mendes EG, Alves JAR, Martins CR. Doença periodontal e cárie dental na população brasileira. Necessidade de tratamento, atenção odontológica e formação profissional. Arq Centro Estudos Curso Odontol. 1977;14(1/2): 61-70.

10. Carvalho de Oliveira AM, Tavares D. Prevalência da cárie dentária em primeiros molares permanentes de escolares novos - zona rural da grande Florianópolis, SC. Rev Fac Odontol UFBa. 1981;1(1):39-55.

11. Makowiecky N, Silva RH. Prevalência de cárie dentária em 277 escolares, alunos novos da $1^{\text {a }}$ série do $1^{\circ}$ grau, de 7 a 10 anos de idade, ambos os sexos, de 35 escolas públicas localizadas na zona rural da região da Grande Florianópolis, estado de Santa Catarina. Rev Catarinense Odontol. 1981;8(2):26-31.

12. Marques DE, Rink MCM, Loureiro RMT, Silva VC. Levantamento epidemiológico de cárie dentária na zona rural de Uberlândia Minas Gerais. Contribuição para um modelo 
de programa de saúde bucal. Rev Centro Cienc Biomed Univ Fed Uberlandia. 1986;2(1): 33-8.

13. Dini EL, Silva SRC. Prevalence of caries and dental care status of schoolchildren from urban and rural areas in Araraquara, SP, Brazil. Int Dent J. 1994;44(6):613-6.

14. Araújo MTB, Araújo RPC, Almeida JCS, Gaudenzi TFB, Campos EJ, Garcia MAS. Aplicação de métodos odontológicos preventivos e curativos nas crianças de faixa etária de 6 a 12 anos, residentes e domiciliadas na área rural do município de Cansanção - Bahia. Rev Baiana Saude Publica. 1994/1995;21(1/4): 9-38.

15. Furtado A, Traebert JL, Marcenes WS. Prevalência de doenças bucais e necessidade de tratamento em Capão Alto, Santa Catarina. Rev ABO Nac. 1999;7(4):226-30.

16. Cangussu MCT, Coelho EO, CastellanosFernández RA. Epidemiologia e iniqüidade em saúde bucal aos 5, 12 e 15 anos de idade no município de Itatiba, São Paulo, 2000. Rev Fac Odontol Bauru. 2001;9(1/2):77-85.

17. Traebert J, Suárez CS, Onofri DA, Marcenes W. Prevalência e severidade de cárie dentária e necessidade de tratamento odontológico em pequenos municípios brasileiros. Cad Saude Publica. 2002;18(3):817-21.

18. Pallos D, Cortelli SC, Oliveira OMS, Ricardo LH, Cortelli JR. Prevalence of DMFT in rural children, young and adult individuals. J Dent Res. 2002;81(Special Issue B):154.

19. Freire MCM, Melo RB, Silva SA. Dental caries prevalence in relation to socioeconomic status of nursery school children in Goiania-GO, Brazil. Community Dent Oral Epidemiol. 1996;24(5):357-61.

20. Moura MS, Pinto LAMS, Giro EMA, Cordeiro RCL. Cárie dentária relacionada ao nível socioeconômico em escolares de Araraquara. Rev Odontol UNESP. 1996;25(1):97-107.

21. Peres KGA, Bastos JRM, Latorre MRDO. Severidade de cárie em crianças e relação com aspectos sociais e comportamentais. Rev Saude Publica. 2000;34(4):402-8.

22. Antunes JLF, Frazão P, Narvai PC, Bispo CM, Pegoretti T. Spatial analysis to identify differentials in dental needs by area-based measures. Community Dent Oral Epidemiol. 2002; 30(2):133-42.

23. Baldani MH, Narvai PC, Antunes JLF. Cárie dentária e condições sócio-econômicas no Estado do Paraná, 1996. Cad Saude Publica. 2002;18(3):755-63.

24. Gonçalves ER, Peres MA, Marcenes W. Cárie dentária e condições sócio-econômicas: um estudo transversal com jovens de 18 anos de Florianópolis, Santa Catarina, Brasil. Cad Saude Publica. 2002;18(3):699-706.

25. Källestal C, Wall S. Socio-economic effect on caries. Incidence data among Swedish 12-14year olds. Community Dent Oral Epidemiol. 2002;30(2):108-14.

26. Tickle M, Milson KM, Blinkhorn A. Inequalities in dental treatment provided to children: an example from UK. Community Dent Oral Epidemiol. 2002;30(5):335-41.

27. Watt RG. Emerging theories into the social determinants of health: implications for oral health promotion. Community Dent Oral Epidemiol. 2002;30(4):241-7.
28. Prefeitura da Cidade de Itaúna. Plano municipal de saúde 2000/2003. Itaúna: Prefeitura Municipal; 2000.

29. Instituto Brasileiro de Geografia e Estatística. Comissão Nacional de Classificação - CONCLA (2002). Disponível em: http://www. ibge.gov.br/concla/default.php. Acessado em 21 de setembro de 2004.

30. Graziano-Silva J. Velhos e novos mitos do rural brasileiro. Disponível em: http://www. grupochorlavi.org/debate/velhosynovos. pdf . Acessado em 21 de setembro de 2004.

31. Brasil, Ministério da Saúde, Secretaria de Políticas de Saúde, Departamento de Atenção Básica, Área Técnica de Saúde Bucal. Projeto SB2000: condições de saúde bucal da população brasileira no ano 2000: manual do examinador. Brasília: Ministério da Saúde; 2001.

32. Organização Mundial da Saúde. Levantamentos básicos em saúde bucal. $4^{a}$ ed. São Paulo: Santos; 1999.

33. Statistical Package for the Social Sciences (SPSS) 11.0 for Windows. Chicago: SPSS Inc.; 2001.

34. Colosimo EA. Análise de sobrevivência aplicada. Piracicaba: ESALQ/USP; 2001.

35. Hosmer DW, Lemeshow S. Applied logistic regression. $2^{\text {a }}$ ed. Nova Iorque: WilleyInterscience Publication; 2000.

36. Oliveira AGRC. Levantamento epidemiológico em saúde bucal - cárie dental. Araçatuba; 1998. Disponível em: http://planeta. terra.com.br/saude/angelonline/artigos/art _epid/epi_96.pdf . Acessado em 21 de setembro de 2004.

37. Santos AMSP. Dinâmica econômica e reorganização espacial. Notas sobre o caso da expansão do capital urbano no espaço rural da Amazônia. Rev Econ Sociol Rural. 1994;32(4): 391-401.

38. Graziano-Silva J, Basadi OV, Bolliger FP, Borin MR, Paro, MR. O rural paulista: muito além do agrícola e do agrário. Sao Paulo Perspect. 1996;10(2):60-72.

39. Chase J. Exodus revisited: the politics and experience of rural loss in Central Brazil. Sociol Ruralis. 1999;39(2):165-85.

40. Borges EM. Programa bolsa-escola de Belo Horizonte: limites e possibilidades de uma política de inclusão social [dissertação]. Belo Horizonte: Escola de Governo/Fundação João Pinheiro; 2003.

41. Oliveira AGRC, Unfer B, Costa ICC, Arcieri RM, Saliba NA. Influência de modificações nos critérios de diagnóstico de cárie nos levantamentos epidemiológicos. Rev CROMG. 1998;4(1):54-60.

42. Chaves SCL, Vieira-da-Silva LM. A efetividade do dentifrício fluoretado no controle da cárie dental: uma meta-análise. Rev Saude Publica. 2002;36(5):598-606.

43. Nörmark S, Mosha HJ. Relationship between habits and dental health among rural Tanzanian children. Community Dent Oral Epidemiol. 1989;17(6):317-21.

44. Carstens IL, Louw AJ, Kruger E. Dental status of rural school children in a sub-optimal fluoride area. J Dent Assoc S Afr. 1995;50(9): 405-11.

45. Gugushe TS, Du Plessis JB. Region urbanrural distribution of dental caries experience in Swaziland. S Afr Dent J. 1998;53(8):40912 .

46. Attin T, Mbiydzemo FN, Villard I, Kielbassa AM, Hellwig E. Dental status of schoolchildren from a rural community in Cameroon. S Afr Dent J. 1999;54(4):145-8.

47. Al-Hosani E, Rugg-Gunn A. Combination of low parental educational attainment and high parental income related to high caries experience in pre-school children in Abu Dhabi. Community Dent Oral Epidemiol. 1998;26(1): 31-6.

48. Li Y, Navia JM, Bian JY. Caries experience in deciduous dentition of rural Chinese children $3-5$ years old in relation to the presence or absence of enamel hypoplasia. Caries Res. 1996; 30(1):8-15.

49. Wong MCM, Lo ECM, Schwarz E, Zhang HG Oral health status and oral health behaviors in Chinese children. J Dent Res. 2001;80(5):145965 .

50. Frazão P. Epidemiologia em saúde bucal. Em: Pereira AC. Odontologia em saúde coletiva. Planejando ações e promovendo saúde. Porto Alegre: Artmed; 2003. Pp. 64-82.

51. Cypriano S, Pecharki GD, Souza MLR, Wada RS. A saúde bucal de escolares residentes em locais com ou sem fluoretação nas águas de abastecimento público na região de Sorocaba, São Paulo, Brasil. Cad Saude Publica. 2003; 19(4):1063-1071. Disponível em: http:// www.scielosp.org/scielo.php?script=sci arttext\&pid $=$ S0102-311X2003000400028 $\& \operatorname{lng}=$ en\&nrm=iso. Acessado em setembro de 2004.

52. World Health Organization. Charte D'Ottawa. La santé de l'homme. 1996;325:3-5.

53. Hausen H, Seppa L, Fejerkov O. A cárie dentária pode ser uma doença previsível? Em: Thylstrup A, Fejerskov O. Cariologia clínica. $2^{a}$ ed. São Paulo: Santos; 1995. Pp. 383-411.

54. Rose G. The strategy of preventive medicine. Oxford: Oxford Medical Publications; 1995.

55. Bezerra ACB. Estudo clínico-epidemiológico da prevalência de cárie em crianças préescolares de 12 a 48 meses de idade [tese]. São Paulo: Universidade de São Paulo; 1990.

56. Holst D, Schuller AA, Aleksejuniené J, Eriksen HM. Caries in populations - a theoretical causal approach. Eur J Oral Sci. 2001;109(3): 143-8.

57. Jamel HA, Sheiham A, Watt RG, Cowell CR. Sweet preference, consumption of sweet tea and dental caries: studies in urban and rural Iraqi populations. Int Dent J. 1997;47(4): 213-7.

58. Lo ECM, Holmgren CJ, Hu D, WAN H. Dental caries status and treatment needs of 12-13 year-old children in Sichuan Province, Southwestern China. Community Dent Oral Epidemiol. 1999;16(2):114-6.

59. Minayo MCS, Assis SG, Deslandes S, Souza ER. Possibilidades e dificuldades nas relações entre ciências sociais e epidemiologia. Cienc Saude Coletiva. 2003;8(1):97-107.

Manuscrito recebido em 3 de março de 2004. Aceito em 20 de julho de 2004. 
ABSTRACT Objective. To study the prevalence of dental caries and its association with socioeconomic variables among schoolchildren living in rural communities in Itaúna, state of Minas Gerais, Brasil, in 2002.

Dental caries Methods. An epidemiological survey was carried out among 476 schoolchildren bein schoolchildren from rural communities in Itaúna tween 4 and 15 years of age. The children underwent a dental examination that was performed by a single researcher following the methodology recommended by the World Health Organization. Socioeconomic data (family income, parents' education and occupation, household source of drinking water, and the child's sex and age) were gathered through an interview with the parents.

Results. Over three fourths of the parents earned no more than 360 reais (R\$) (approximately US\$153) a month and reported having 4 years of schooling at most. Considering both the deciduous and permanent dentitions, $17.86 \%$ of the children were free of caries. The mean number of permanent teeth that were decayed, missing or filled, and of primary teeth that were filled or decayed and needed extraction, was $0.94( \pm 1.55)$ and $4.00( \pm 3,46)$, respectively. Multivariate analysis showed that among seven- and eight-year-old children whose monthly family income was above $\mathrm{R} \$ 280$, the risk of having caries in the primary (deciduous) dentition and in both dentitions was 2.602 (95\% CI: 1.004 to 6.745) and 2.854 (95\% CI: 1.044 and 7.799) times greater, respectively, than among children from lower-income families. Seven- and eightyear-old children whose mothers had completed at least 3 years of elementary school showed more than twice the risk [2.813 (95\%CI: 1.221 to 6.480$)$ ] of having caries in the permanent dentition than children whose mothers had less schooling.

Conclusions. In view of these results, particularly those pertaining to the primary dentition, there is an urgent need to implement community dental health strategies targeting young children in Brasil. The results suggest that in underprivileged populations, such as the inhabitants of rural communities in Itaúna, families with somewhat higher incomes and better schooling are more likely to adopt behaviors that lead to dental caries.

The only way for a rich man to be healthy is by exercise and abstinence, to live as if he were poor.

[Para el hombre rico, la salud se consigue de una sola manera: mediante el ejercicio y la abstinencia, es decir, viviendo como si fuese pobre.]

Paul Dudley White

U. S. cardiologist / cardiólogo estadounidense 